\title{
An Exploration of Pen Rolling for Pen-based Interaction
}

\author{
Xiaojun $\mathrm{Bi}^{1}$, Tomer Moscovich ${ }^{1}$, Gonzalo Ramos ${ }^{2}$, Ravin Balakrishnan ${ }^{1}$, Ken Hinckley ${ }^{3}$ \\ ${ }^{1}$ Department of Computer Science \\ University of Toronto \\ www.dgp.toronto.edu \\ xiaojun, tomer, ravin@ dgp.toronto.edu \\ ${ }^{2}$ Microsoft Live Labs \\ Washington, USA \\ labs.live.com \\ gonzalo@microsoft.com \\ ${ }^{3}$ Microsoft Research \\ Washington, USA \\ www.research.microsoft.com \\ kenh@ microsoft.com
}

\begin{abstract}
Current pen input mainly utilizes the position of the pen tip, and occasionally, a button press. Other possible device parameters, such as rolling the pen around its longitudinal axis, are rarely used. We explore pen rolling as a supporting input modality for pen-based interaction. Through two studies, we are able to determine 1) the parameters that separate intentional pen rolling for the purpose of interaction from incidental pen rolling caused by regular writing and drawing, and 2) the parameter range within which accurate and timely intentional pen rolling interactions can occur. Building on our experimental results, we present an exploration of the design space of rolling-based interaction techniques, which showcase three scenarios where pen rolling interactions can be useful: enhanced stimulus-response compatibility in rotation tasks [7], multi-parameter input, and simplified mode selection.
\end{abstract}

ACM Classification: H5.2 [User Interfaces]: Input devices and strategies

General terms: Design, Human Factors

Keywords: Input devices, multi degree of freedom input, rotation, pen

\section{INTRODUCTION}

Pen input is emerging as a promising interaction modality for slate computers, handheld devices, and large format electronic whiteboards. Typically, only the x-y movements of the pen are sensed and used for interaction but some devices, such as Tablet PCs and desktop digitizing tablets, can provide additional degrees of freedom including pressure, tilt, and rolling around the pen's longitudinal axis.

Although rolling as input has initially been explored by Miura et al. [16] and Suzuki et al. [20], its properties and potential as an additional input modality have not been fully investigated. Adding an auxiliary stream to an input device

Permission to make digital or hard copies of all or part of this work for personal or classroom use is granted without fee provided that copies are not made or distributed for profit or commercial advantage and that copies bear this notice and the full citation on the first page. To copy otherwise, or republish, to post on servers or to redistribute to lists, requires prior specific permission and/or a fee.

UIST'08, October 19-22, 2008, Monterey, California, USA.

Copyright 2008 ACM 978-1-59593-975-3/08/10 ...\$5.00. has been shown to significantly improve performance [23], with the most compelling example being mouse scrollwheels [10]. Nowadays, scroll wheels are incredibly useful across the GUI and have become ubiquitous. Thus, it is worth exploring if utilizing an additional degree of freedom such as pen roll could improve pen interaction in much the same way scroll wheels have improved mouse interaction, particularly in a "pure pen computing" scenario where additional devices such as mice and keyboards are absent.

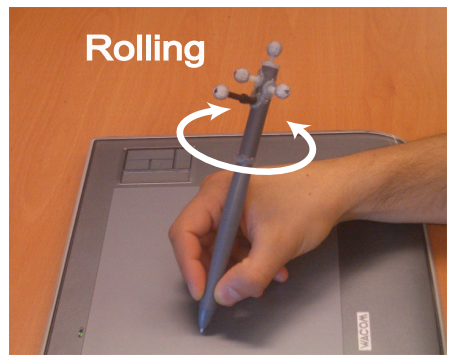

Figure 1. Rolling a pen.

Unlike a mouse wheel which is relatively independent of other mouse degrees of freedom, pen rolling is closely linked to the pen's other degrees of freedom and can occur incidentally during the course of normal pen use. Such incidental rolling and interrelationships between the pen's degrees of freedom are not yet well understood, which makes it difficult to design interfaces utilizing pen rolling.

In this paper, we investigate the properties of rolling a pen from a human performance standpoint, and begin to explore the design space of rolling-based interaction techniques. We conduct two experiments that quantify the characteristics of rolling the pen around its longitudinal axis (while holding it in a traditional pen grip-Figure 1). Experiment 1 investigates incidental pen rolling caused by regular drawing and writing, in order to determine thresholds for separating such events from intentional rolling used for interaction, while experiment 2 quantifies parameter ranges where accurate and timely intentional pen rolling takes place. Based on the result of these experiments, we implement prototype applications that incorporate rolling into pen interaction. These showcase three scenarios of utilizing pen rolling: enhanced stimulus-response compatibility in graphical rotation tasks [7], multiparameter input, and simplified mode selection. 


\section{RELATED WORK}

Although roll-aware styli are available for digital painting applications [1], rolling as input has only been investigated by a few researchers. Miura and Kunifuji [16] explore this modality by suggesting that a pen may act as a knob while stowed in an instrumented holder on a portable device. Suzuki et al. [20] use an accelerometer to detect pen rolling, and used roll to select a drawing color or scrolling up/down.

Other methods have been considered for enhancing pen input. Pen pressure has been demonstrated to be a viable mode of auxiliary input [18]. Pressure has also long been used to control brush size in drawing software, and is the basis of a variety of interaction techniques $[18,19]$. Pen tilt has also been used for interaction: matching cursor shape to tilt angles can improve performance by enhancing stimulusresponse compatibility [21].

Some input devices provide parallel streams of input. These are frequently designed for specific tasks requiring simultaneous control of multiple degrees of freedom. Zhai et al. [23] investigate positioning and orienting objects in three-dimensional space with a six degree of freedom input device. Liu et al. [13] use a cylindrical "block" to simultaneously rotate and translate objects on digital tables.

Truly parallel input is possible but not always achievable or desirable. Many interaction techniques are crafted around carefully combining degrees of freedom in just the right way in an attempt to make the interactions naturally correspond to the desirable movements while remaining controllable and stable. This type of stability is most easily achieved by multi-channel input devices which provide easily separable channels of interaction [14]. Typically, one of these channels serves as a primary means of input (e.g. cursor control), while secondary channels act in a supporting role (e.g. scrolling). Mice augmented with a wheel and joysticks are common examples [24]. Mice that can detect rotation and tilt have also been designed [4].

An additional stream of input can also be controlled by the user's non-dominant hand. An everyday example is that of a user operating a mouse in the right hand, while executing keyboard commands with the left. Use of the non-dominant hand in a task that supports the primary manipulation of the dominant hand has been shown to improve performance for scrolling and graphical object manipulation $[5,10]$.

While the degrees of freedom of a pen are somewhat coupled, there is evidence that their control can be separated [22]. Human movement research shows that holding a pen in a regular writing grip (Figure 1) can be classified as a type of precision grasp, which allows users to perform delicate tasks with low power $[15,17]$. However, there is little data in the literature as to the parameters governing human ability to control rolling; this provides motivation for our current work which systematically studies pen roll properties, and explores rolling based interaction techniques.

\section{EXPERIMENT 1: INCIDENTAL ROLLING}

Pen rolling can occur incidentally when a user is drawing or writing. In contrast, rolling-based interaction techniques require a user to intentionally roll a pen. The challenge of avoiding interference between incidental and intentional rolling is mentioned by Suzuki et al. [20]. In their work, a $10^{\circ}$ difference in rotation angle over $100 \mathrm{~ms}$ is used to discriminate intentional from incidental rolling.

To more effectively distinguish these two types of rolling actions, we quantitatively investigate the properties of incidental pen rolling. In addition, we investigate the effects of handedness on pen rolling, to determine whether we should take handedness into account when classifying rolling as intentional or incidental.

\section{Apparatus}

The experiment (Figure 2) was conducted with an 18-inch LCD monitor running at a native resolution of $1280 \times 1024$ pixels. The experimental software ran on a $3 \mathrm{GHz} \mathrm{PC}$ with Windows XP. The pen operated on a Wacom Intuos $36 \times 11$ digitizing tablet $(15.2 \times 27.9 \mathrm{~cm})$ offering a resolution of 5080 lines per inch (200 lines per $\mathrm{mm}$ ). The tablet's active area was mapped onto the entire display area, and pen events were sampled at $50 \mathrm{~Hz}$. An 8 camera Vicon motion tracking system [2] was used to determine the rolling angle of the pen, which had four passive reflective markers placed on it. These four small makers add $2 \mathrm{~g}$ to the pen that weighed $14 \mathrm{~g}$ originally. All the markers are attached above the user's hand, at a minimum distance of $15 \mathrm{~cm}$ from the tip of the pen. Although this asymmetrically dispersed mass may affect the pen balance, no participant commented on the imbalance; hence, we believe that the markers have negligible effect on the feel of the pen. As with pen events, tracking data was streamed into the applications at $50 \mathrm{~Hz}$. Pen rolling angles were calculated based on the 3D positions of these markers. Note that we used the Vicon system as it provides accurate tracking which ensures that we collect the best possible data in this experimental setting; a real application would instead rely on the tablet hardware (e.g., Wacom 6D Art Pen [1]) for roll detection at perhaps a slightly reduced fidelity.
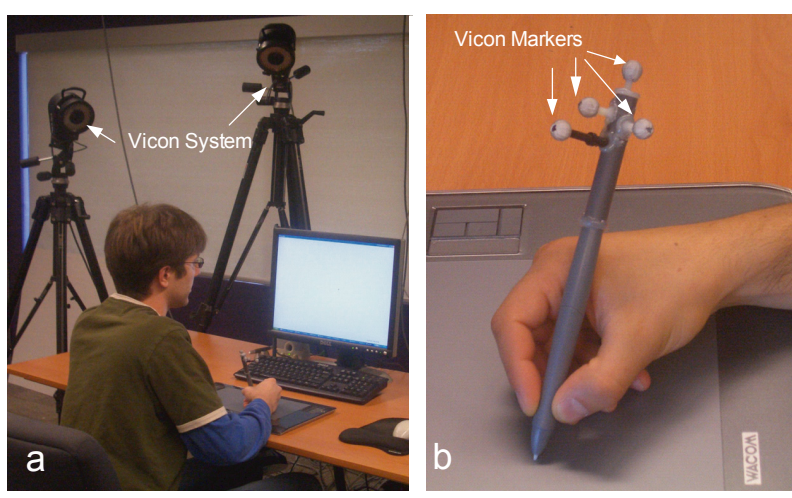

Figure 2. (a) Experiment setup. (b) A user holds the tracked Wacom Ink pen with attached Vicon markers. 


\section{Participants}

Eight volunteers (2 female, 6 male), 18-34 years old, participated. Four were left-handed. One right-handed and one left-handed participant used a pen based device about once a week, and others used it about once a month. They were not told that we were investigating pen rolling.

\section{Tasks}

The experiment consisted of four tasks:

1. Free Drawing. Participants copied line drawings printed on individual sheets of paper

2. Writing. Participants wrote short sentences on the tablet. These were also copied off printed sheets.

3. Screen Tracing. Pictures were displayed on the screen and participants were asked to trace them using the pen.

4. Tablet Tracing. Printed drawings were placed on the tablet, and participants traced them using the pen.

\section{Design}

The four left-handed and four right-handed participants were assigned to two groups respectively. In each group, participants performed all four tasks, whose order of presentation was counterbalanced using a Latin square. For each task, participants completed 8 trials (i.e. 8 pages of writing, drawing, screen tracing, or tablet tracing) for a total of 32 trials for the four tasks. Presentation of pages within a task was randomized. Prior to each task, participants were given two warm-up pages to familiarize themselves with the task. Participants were instructed to perform the task as quickly and accurately as possible. A two minute break was enforced between tasks. The experiment lasted approximately 1.5 hours for each participant.

\section{Measures}

We define a stroke to be pen tip movement occurring between a tip's touch-down and subsequent lift-up events. For each sample point along a stroke, we compute two dependent variables: rolling angle and rolling speed.

Rolling angle is defined as the amount of rotation around the pen's longitudinal axis that occurs between the start point of the stroke and the current sample point, with a positive value corresponding to a clockwise rotation. For example, a 15 degree rolling angle indicates 15 degree clockwise rotation around the pen's longitudinal axis between the current sample point and the start of a stroke.

Rolling speed is simply the difference in rolling angle between current sample point and the previous one, divided by the interval between them $(\Delta \mathrm{t})$. It represents how fast a user rolls the pen between consecutive sample points. As rapid rolling actions could play a key role in rolling based interaction, it is important to see if they occur incidentally.

To estimate the measurement accuracy of our system, we measured the background noise of our experimental setup by fixing the pen at 0,45 , and 90 degrees, and recording the rolling angle and rolling speed of the pen for 10 seconds at each position. The standard deviation of rolling angle was 0.03 degrees while the standard deviation of rolling speed was 1.5 degree/s with a mean of 0 .

\section{Results of Experiment 1}

Figure 3 shows our measures broken down by task. Rolling speed was fastest in the writing task, but slowest in the free drawing task. Writing consists of controlled movements which can be performed quickly. These quick hand and finger movements may cause fast pen rolling. In contrast, drawing and tracing are closed-loop steering tasks [3], which are performed slowly and probably more carefully

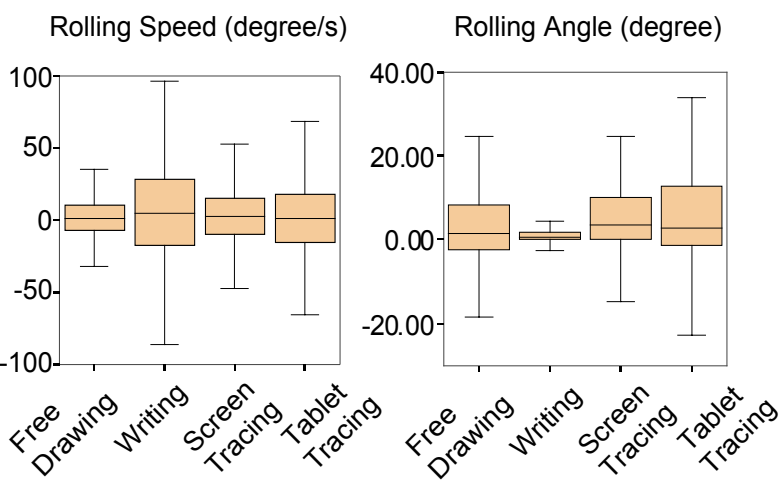

Figure 3. Measures by task: (a) rolling speed, (b) rolling angle.

As shown in Figure 4. rolling angle increases with the distance from the start point of a stroke (Pearson correlation coefficient $\mathrm{r}=0.30, \mathrm{p}<.01$ ) with an overall tendency for clockwise rotation. The mean rolling angle for all data is $3^{\circ}$ $\left(\mathrm{SD}=10^{\circ}\right)$ with no significant difference between right- and left-handed participants (Independent t-test, $\mathrm{t}(6)=0.86, \mathrm{p}=$ 0.42 ). This tendency towards a positive rolling angle may be a product of the left-to-right, top-to-bottom scanning order of writers of English. The rolling angle of a stroke appeared to be related to whether the stroke was drawn clockwise or counterclockwise. Specifically, the signedarea of strokes (which indicates the clock direction a path is drawn) is slightly correlated with the rolling angle (Pearson correlation $\mathrm{r}=0.12, \mathrm{p}<.01)$. We find this relationship holds across all tasks (Figure 4 ).

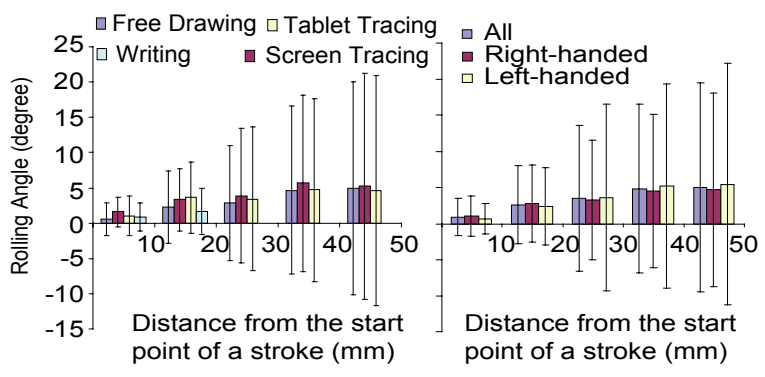

a)

Figure 4. Mean and standard deviation of rolling angle by (a) task (note that all the data in the writing task was below 20 mm), (b) handedness. 
Figure 5 illustrates the distribution of rolling speed broken down by handedness. Again, the distributions of right- and left-handed participants exhibit no significant difference (Independent t-test, $\mathrm{t}(6)=0.49, \mathrm{p}=0.64$ ). As may be expected from the rolling angle data, more rolling occurs in the clockwise direction, regardless of handedness. The mean rolling speed is $3 \% \mathrm{~s}$. Disregarding the direction of rolling, the mean rolling speed is $20 \%$, with no significant difference between the right and left-handed groups (Independent t-test, $\mathrm{t}(6)=0.29, \mathrm{p}=0.78$ ).

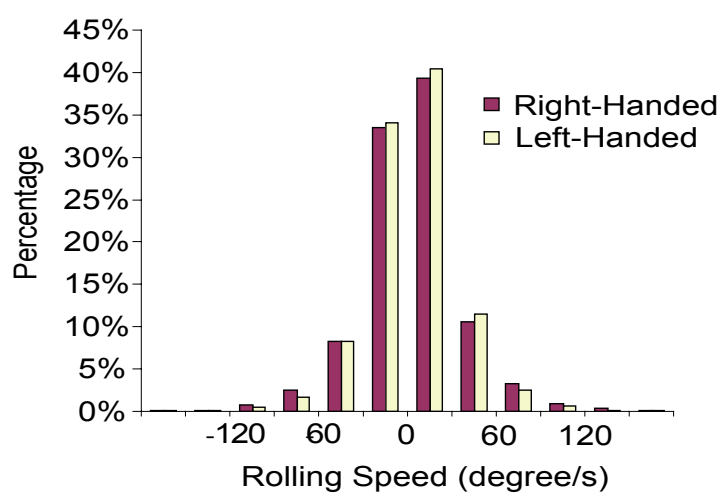

Figure 5. Distribution of rolling speed by handedness

\section{Discussion of Experiment 1}

Incidental vs. Intentional Rolling

Building on the experiment results, we can characterize incidental pen rolling by rolling speed and rolling angle. We found that $74.1 \%$ of the data has a rolling speed in the $[-30,30]$ degree/s range, and that $77.5 \%$ has a rolling angle in the $[-10,10]$ degrees range. If we consider both of these variables together, we find that $98.1 \%$ of the data can be characterized as being either in the rolling speed range of [$30,30]$ degree/s or in the rolling angle range of $[-10,10]$ degrees. These short ranges of rolling speed and rolling angle suggest that a user does not roll a pen severely when performing typical drawing and writing tasks. Furthermore, we observed during the experiment that there were cyclical changes, clockwise followed by counterclockwise rotations, which may contribute to the small range of rolling angle.

Based on the experimental data, we can identify a pen event as incidental rolling if it falls within the rolling speed range of $[-30,30]$ degree/s or the rolling angle range of $[-10,10]$ degrees, indicating it is a consequence of typical pen use. In contrast, when rolling a pen on purpose for interaction, we expect that a user would roll the pen faster and with a bigger angle compared to incidentally rolling a pen in regular drawing and writing. Thus, rolling motion falling outside both of these ranges can likely to be classified as intentional rolling, and used for purposeful rolling actions performed by the user for explicit interaction tasks that are complementary to the flow of users' existing pen-based drawing and writing tasks.

\section{The Effect of Handedness}

Measures for right- and left-handed participants were nearly identical-even with respect to rolling direction. Although 3 out of 4 experimental tasks involved drawing and not writing per se, we suspect this result might partially be due to users' prior experience with writing English, where writing is done from left to right, top to bottom, regardless of handedness. Furthermore, even when holding the pen in the same hand, different users may use very different grips. For example, some participants held the pen between their thumb and index fingers, while others held it between their index and middle fingers. The differences caused by the various grips may outweigh the differences caused by handedness. Based on these results, it appears that handedness need not be taken into account when classifying rolling as incidental or intentional.

\section{EXPERIMENT 2: INTENTIONAL ROLLING}

This experiment investigates user ability to control intentional rolling. In particular, we are concerned with the usable range within which a user can comfortably roll a pen; and the easily discriminable rotation that a user can intentionally specify quickly and accurately with a pen. In addition, we also investigate the co-variation between rolling and $\mathrm{x}-\mathrm{y}$ position of the pen tip - a key element in determining effective ways to integrate rolling with existing pen interfaces that rely on $\mathrm{x}-\mathrm{y}$ position of the pen tip.

\section{Apparatus and Participants}

The hardware is the same as in Experiment 1. Twelve volunteers ( 3 female, 9 male), 18-35 years old, participated None participated in Experiment 1. Four were left-handed. Eight used a pen-based device about once a month, and others had no prior experience with such devices.

\section{Task and Procedure}

We used a serial targeting task. Figure 6 shows how a trial begins by moving the cursor into the starting circle in the centre of the screen using the pen. A blue circular range sector appears, indicating the angular distance (D) to the target. Along the vertical axis of the range sector, a red rolling line is displayed, while on the other side a green target sector is shown. The rolling line rotated as the users rolled the pen around its longitudinal axis. Once the pen touched the tablet, the rolling angle of the pen was mapped to the rotation of the rolling line in a one-to-one mapping. For example, one degree rolling angle rotates the rolling line one degree clockwise. The participant's objective is to rotate the rolling line into the target sector and lift the pen tip up to confirm the selection. The target sector is drawn in yellow whenever the rolling line is within it, providing visual feedback to the user similar to the highlighting feedback that is ubiquitous in modern interfaces. Should the user fail to select the target (e.g. by lifting the pen with the rolling line outside the target), the system plays a "beep" sound to indicate the error. Both the angular width of the target (W) and the angular distance (D) to this target were manipulated as independent variables across trials. 


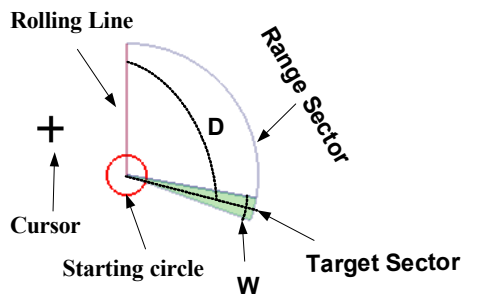

a)

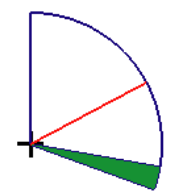

c)

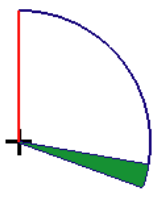

b)

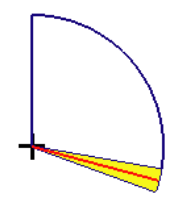

d)
Figure 6. Task for experiment 2. (a) The user moves the cursor into the starting circle; (b) the trial begins; (c) the user rolls the pen to rotate the rolling line into the target sector; (d) the target is highlighted to indicate selection.

\section{Design}

A within-participants full factorial design with repeated measures was used. The independent variables are the angular distance of the range sector (D), representing the usable range, and angular width of the target sector (W), which represents the easily discriminable rotation. To determine the usable range, we test ten values of $\mathrm{D}$ distributed along a full revolution $\left(\mathrm{D}=-180^{\circ},-135^{\circ},-90^{\circ}\right.$, $\left.50^{\circ},-10^{\circ}, 10^{\circ}, 50^{\circ}, 90^{\circ}, 135^{\circ}, 180^{\circ}\right)$. To home in on the easily discriminable rotation, we test $\mathrm{W}$ at $3^{\circ}, 10^{\circ}$ and $15^{\circ}$. These three values represent small, medium and large angles respectively, determined through pilot testing. The $\mathrm{W}$ values were fully crossed with all $10 \mathrm{D}$ values, resulting in 30 conditions. Each participant completed 5 blocks of trials, with each block consisting of all 30 W-D combinations appearing 5 times each in random order.

Prior to performing the trials, the experimenter explained the task to participants, who then completed a 20-trial warm-up block. Participants were instructed to perform the trials as accurately and quickly as possible, and were not required to limit tip movement (i.e., we wanted the user to use the pen in a way that might resemble real pen usage). A 3 minute break was enforced between blocks, with the experiment lasting approximately 100 minutes for each participant. A short questionnaire was administered at the end of the experiment to gather subjective opinions. In questionnaires, participants were asked to label each D and W value as either "comfortable" or "uncomfortable" for rolling according to their experience in the experiment

\section{Measures}

We computed four dependent variables per trial:

Selection time, defined as the time from the moment the pen touches the tablet's surface until its tip leaves the tablet.

Number of crossings (NC), defined as the number of times the rolling line entered or left the target sector per trial (e.g., this value is 1 if the user did not overshoot the target sector and 3 if the user overshot once). This measure indicates the degree of control exhibited by users.

Error rate (ER), defined as the percentage of trials for a particular condition that resulted in erroneous selections.

Tip movement, defined as the distance the pen tip travels from the moment the pen touches the tablet's surface until its tip leaves the tablet.

\section{Results of Experiment 2}

Figure 7 illustrates the mean results for selection time, number of crossings, and error rate for Experiment 2.

\section{Easily Discriminable Rotation}

Analysis of variance showed a significant main effect of $\mathrm{W}$ on selection time $(\mathrm{F}(2,22)=321.4 \mathrm{p}<0.0001)$, with significant differences between all pairs of target widths $(\mathrm{p}$ $<0.001)$. The greatest incremental difference was between

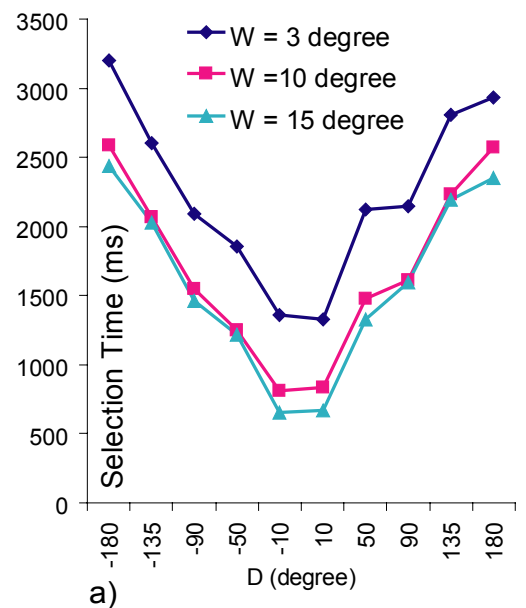

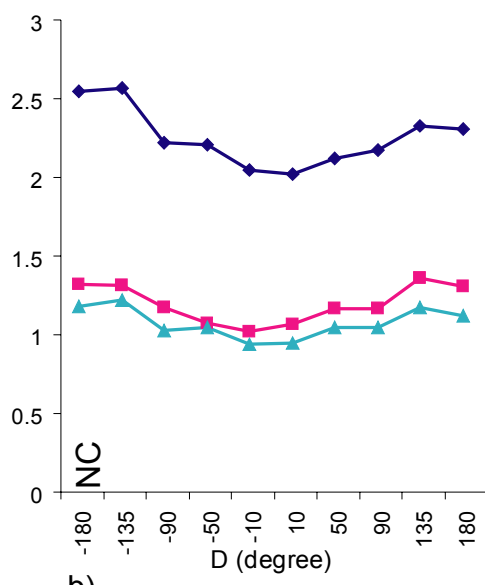

b)

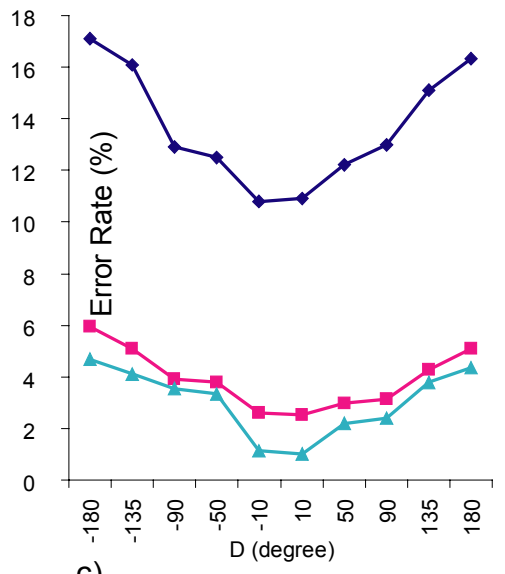

c)

Figure 7. Mean Results for Experiment 2. (a) Selection time, (b) Number of crossings, (c) Error rate. 
$\mathrm{W}=10^{\circ}$ and $\mathrm{W}=3^{\circ}$, with a mean of 0.663 seconds.

There was a significant main effect of $\mathrm{W}$ on number of crossings $(\mathrm{F}(2,22)=201.5, \mathrm{p}<0.0001)$. While significant differences were found for all pairs, the greatest loss of control occurred between $\mathrm{W}=10^{\circ}$ and $\mathrm{W}=3^{\circ}$, where the mean number of crossings jumped by 1.05 .

There was also a significant main effect of $\mathrm{W}$ on error rate $(\mathrm{F}(2,22)=201.5, \mathrm{p}<0.0001)$. As with selection time and number of crossings, all pairs were significantly different, with the largest difference between $\mathrm{W}=10^{\circ}$ and $\mathrm{W}=3^{\circ}$.

From this analysis, we see that user performance measured by selection time, number of crossing and error rate deteriorates as $\mathrm{W}$ decreases. In particular, the largest degradation in performance occurs for $\mathrm{W}$ between $10^{\circ}$ and $3^{\circ}$. Because of this we identify easily discriminable rotations as the ones where $\mathrm{W}>10^{\circ}$.

\section{Usable Range}

Analysis of variance showed a significant main effect of D on selection time $(\mathrm{F}(9,99)=412.5, \mathrm{p}<0.0001)$, number of crossings $(\mathrm{F}(9,99)=135.4, \mathrm{p}<0.0001)$, as well as error rate $(\mathrm{F}(9,99)=142.1, \mathrm{p}<0.0001)$. Interestingly, selection time was symmetric along clockwise and counterclockwise rotations, with no significant differences found between corresponding angles. As expected from Fitts' law [6], selection time increases as the range sector (D) grows. The greatest incremental difference was between $\mathrm{D}=90^{\circ}$ and $\mathrm{D}$ $=135^{\circ}$, and the second greatest one was between $\mathrm{D}=-90^{\circ}$ and $\mathrm{D}=-135^{\circ}$. Number of crossings increased significantly with distance, leveling off at $135^{\circ}$ and $-135^{\circ}$, while error rates also increased significantly at angular distances greater than $90^{\circ}$. These results demonstrate that user performance deteriorates as the range sector (D) is enlarged. In particular, the performance deteriorates dramatically when $\mathrm{D}>90^{\circ}$ or $\mathrm{D}<-90^{\circ}$. Thus, we can reasonably use $\left[-90^{\circ}, 90^{\circ}\right]$ as the usable range.

\section{Subjective Opinions}

Figure 8 presents the results of post-experiment questionnaires. All eight participants labeled $\mathrm{W}=10^{\circ}, \mathrm{W}=$ $15^{\circ}$ "comfortable", but $\mathrm{W}=3^{\circ}$ "uncomfortable". Among the $10 \mathrm{D}$ values, all eight participants labeled $-90^{\circ},-50^{\circ}$, $10^{\circ}, 10^{\circ}, 50^{\circ}, 90^{\circ}$ "comfortable", while only two of them labeled $\mathrm{D}=-135^{\circ}$ and $\mathrm{D}=135^{\circ}$ "comfortable". None of participants labeled $\mathrm{D}=-180^{\circ}$ or $\mathrm{D}=180^{\circ}$ "comfortable". Participants reported that it was easy to roll a pen for the angles they labeled "comfortable", since it requires only minor movements of the thumb and index fingers. However, they felt physical discomfort for rolling angles they labeled "uncomfortable".

These subjective scores are in agreement with the previously estimated easily discriminable rotation and usable range, i.e., users felt that they could "comfortably" perform rolling with $\mathrm{W}>10^{\circ}$ and $-^{\circ} 90<\mathrm{D}<90^{\circ}$.

\section{Co-variation between Rolling and Tip Movement}

As shown in Figure 9, as the range sector (D) becomes larger, the pen tip travels a longer distance. Furthermore, analysis of variance shows a significant main effect of $D$ on tip movement $(\mathrm{F}(9,99)=98.4, \mathrm{p}<0.0001)$. We found no significant main effect on tip movement for $\mathrm{W}$, nor any $\mathrm{D} \times$ $\mathrm{W}$ interaction. When confined to the usable range $\left[-90^{\circ}\right.$, $90^{\circ}$, the mean incidental tip-movement was less than $5 \mathrm{~mm}$.

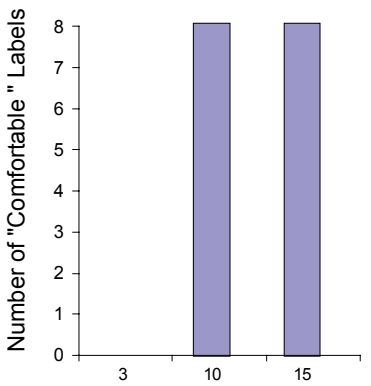

W (degree)

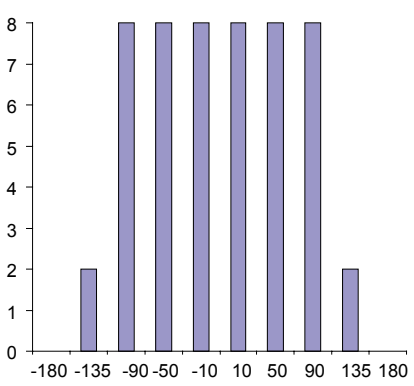

D (degree)
Figure 8. Number of "comfortable" labels by W and D.

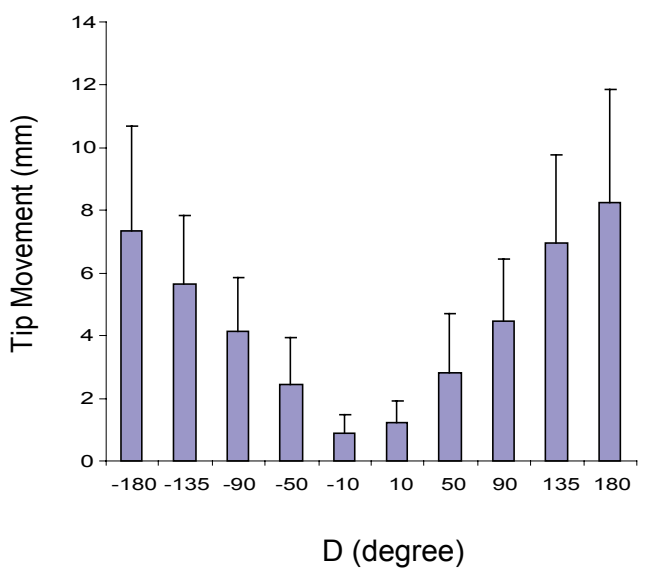

Figure 9. Mean and standard deviation of tip movement by $D$.

\section{Discussion of Experiment 2}

According to the quantitative measures (selection time, number of crossings, error rate) and subjective opinions, we estimated the easily discriminable rotation to be $\mathrm{W}>$ $10^{\circ}$ and the usable range to be $\left[-90^{\circ}, 90^{\circ}\right]$. Within these ranges, users can effectively control intentional rolling: selection time is less than 1.5 seconds; number of crossings is less than 2; error rate is less than $4 \%$; and users are "comfortable" when performing rolling actions. With angles outside these ranges, user performance deteriorates dramatically and users may feel discomfort.

As the participants were not required to limit tip movement when performing rolling, we were able to investigate the co-variation between rolling and the incidental pen tip movement. This knowledge could guide UI designers to effectively incorporate intentional rolling interaction techniques into existing pen interfaces without significantly affecting drawing and writing operations. Our results 
showed a co-variation between rolling actions and pen tip movement. A bigger rolling angle could lead to a greater pen tip movement. In particular, if the rolling angle is within our empirically determined usable range (i.e., $\mathrm{D} \in[-$ $\left.\left.90^{\circ}, 90^{\circ}\right]\right)$, the mean of tip movement is less than $5 \mathrm{~mm}$. Thus, we suggest that if $>5 \mathrm{~mm}$ pen tip movement is acceptable in applications, pen rolling can be used in parallel with $x-y$ tip movement (i.e., a user can roll the pen and move the pen tip simultaneously to interact); if $<5 \mathrm{~mm}$ movement is critical, pen rolling should be utilized separately from tip movement, to avoid interference between these two input channels.

Note that it is certainly also worthwhile to investigate covariation between rolling with other input channels, like pressure, and tilting. However, since most pen-based applications currently only utilize $\mathrm{x}-\mathrm{y}$ tip movement and our focus is on adding rolling to these $x-y$ pen interactions, these co-variations with other pen degrees of freedom are beyond the scope of this paper.

\section{ROLLING BASED INTERACTIONS}

Building on the experiments, we begin an exploration of the design space of rolling based interaction techniques. We implement prototype applications which incorporate pen rolling into pen-based interfaces, showcasing three potential advantages of rolling-based interactions: enhanced stimulus-response compatibility [7] in rotation tasks, multiparameter input, and simplified mode selection.

To avoid interference with regular pen drawing and writing, rolling-based interaction techniques are only activated by intentional pen rolling, with subsequent rolling used for interaction. This allows for normal use of a pen that is not affected by incidental rolling. Lifting the pen tip up terminates the rolling interaction. We use the threshold ranges determined in experiment 1 to separate intentional from incidental pen rolling. If the rolling speed of a data event is between $-30 \%$ and $30 \%$ or the rolling angle is between $-10^{\circ}$ and $10^{\circ}$, we identify it as incidental rolling.

Building upon the results of Experiment 2, we select $-90^{\circ}$ to $90^{\circ}$ as the usable range of rolling. Our prototype applications use the rolling angles only within this range.

The rolling cursor (Figure 10) is designed to provide feedback on the rolling angle. By default, the system depicts the simple version of rolling cursor (Figure 10a), to reduce clutter, and to indicate that rolling interaction is not activated. The rolling line shows the actual rolling angle, and is reset to zero each time the pen leaves the tablet.

Once rolling-based interaction is activated, the full rolling cursor (Figure 10b) fades in, while the simple rolling cursor fades out. The full rolling cursor consists of two $90^{\circ}$ sectors, corresponding to the usable range of rolling. When the user lifts the pen tip, rolling interaction techniques are deactivated and the simple cursor is shown again.

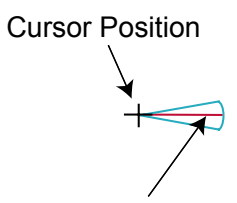

Rolling Line

(a)

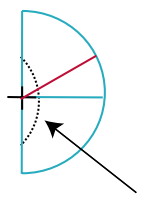

Usable Range $\left[-90^{\circ}, 90^{\circ}\right]$

(b)
Figure 10. (a) Simple rolling cursor, (b) Full rolling cursor

\section{Rolling for Rotation Tasks}

Rotating an object is a common task in drawing and animation applications. However, if the interface relies only on the position of the pen tip, performing object rotations requires separate steps: first selecting the rotation center, and then dragging a corner of the object to execute (e.g., as in Microsoft Office Visio). Employing pen rolling allows a user to rotate an object in a more fluid manner. After selecting the rotation center with position of the pen tip, the user directly rotates the object by rolling the pen: clockwise rolling of the pen produces clockwise rotation of the object, and similarly for counter-clockwise rolling. By combing rolling with $x-y$ tip movement, the object rotates and moves as if attached to the pen tip. As rolling is essentially a rotation action, the system provides a consistent relationship between perception (object rotation) and action (pen rolling), ensuring a high degree of stimulus-response compatibility [7]. We implement two applications that show using pen rolling to rotate a puzzle piece (a), and animate a graphic (b) .

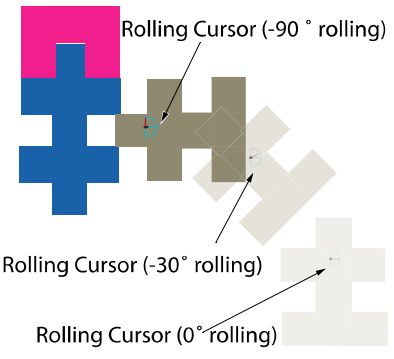

(a)

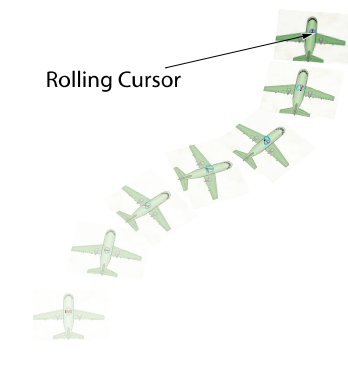

Figure 11. (a) Rotating a puzzle piece by pen rolling and moving it with the pen tip. The rolling cursor indicates the pen tip position as well as the rotation center of the selected object; (b) Rotating and moving a graphic by rolling and moving the pen.

\section{Multi-parameter input}

The mouse wheel is arguably a very useful invention which has reached the status of being taken for granted. It successfully increases the interaction bandwidth of a mouse. However, when exclusively using a pen to interact, users lose the benefits provided by the mouse wheel. Since the typical pen-based interface relies only on the tip position, it cannot simulate the functionality of a mouse wheel. Various methods have been explored to enhance a 
pen's interaction bandwidth, and make it as effective as a mouse. Pen tip pressure has been demonstrated to be a viable input channel [18], however, it is effectively a onedirectional input modality as it can only be manipulated from zero to some maximally sensed pressure value and back to zero again; negative pressure beginning at zero is not possible. Pen rolling, in contrast, can be controlled in two directions from the zero position, and has the potential to be for pens what a scroll wheel is for mice, as initially explored by Suzuki et al. [20].

Associating pen rolling with mouse wheel behavior enables a pen user to access a variety of controls already designed for mouse wheel use. Several examples are illustrated in Figure 12. In a map navigation task (Figure 12a), panning is controlled by pen tip movement and zooming is controlled by pen rolling. Since panning and zooming are controlled by separate input channels, a user can pan and zoom simultaneously. When reading a document, a user can make annotations with the pen tip, and then immediately scroll down or up by rolling the pen (Figure 12b). This technique, similar to that demonstrated by Suzuki et al. [20], allows a user to navigate a document without any pen tip movement, thus maintaining interaction focus on the point of interest. Combining rolling with both tip pressure and tip position allows a user to control four degrees of freedom. This can benefit high-degree-of-freedom control tasks, such as curve editing. As illustrated in Figure $12 \mathrm{c} \& d$, given a control point of a curve, a user can change its position by moving the pen tip, adjust the tangent direction by rolling the pen, and increase the tangent's magnitude by applying more tip pressure.

\section{Mode Selection}

Mode selection presents a usability challenge for pen-only systems where a keyboard or mouse is not available [12]. A typical barrel button can only distinguish between two states, and is not practical in many situations where one might need to select between several modes. The typical solution of having an on-screen tool-palette for mode selection suffers from the "round-trip travel" problem [8], which slows users down and diverts visual attention from their primary task.

Pen rolling can provide a localized, one-handed, and button-free approach for mode switching by mapping different rolling angles and directions to corresponding modes. For example, in a drawing application (Figure 13), rolling angle ranges $\left[-90^{\circ},-45^{\circ}\right],\left[-45^{\circ}, 0\right],\left[0,45^{\circ}\right]$, and $\left[45^{\circ}, 90^{\circ}\right]$ are associated with an eraser, a brush, a pen, and a flood-fill tool, respectively. A user selects a specific drawing tool by rolling the pen to the corresponding angle. Another example is selecting a drawing color by rolling the pen, which is demonstrated by Suzuki et al. [20]. Pen rolling allows a user to select modes without moving the pen tip, eliminating the round-trip travel and maintaining focus on the task at hand.

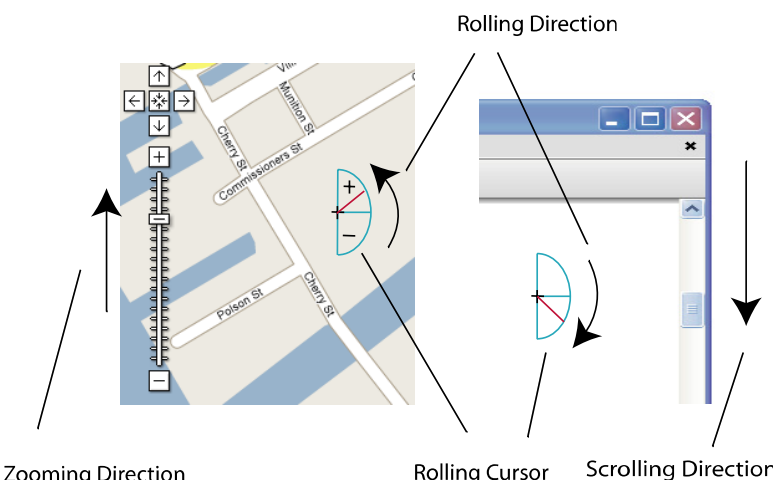

Zooming Direction

(a)

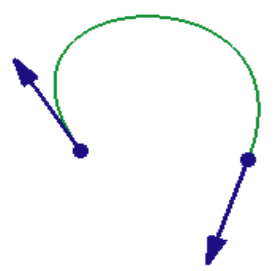

(c) (b)

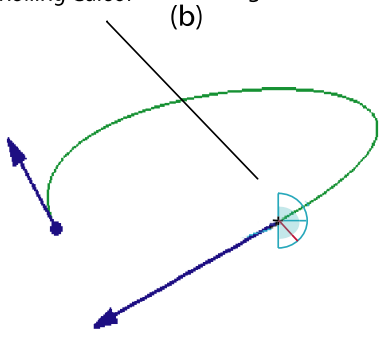

(d)
Figure 12. Example uses of pen roll for multi parameter input. (a). Using pen rolling to control zoom factor in a map navigation application. Counter clock-wise rolling is mapped to zooming in. (b). Rolling a pen to scroll down/up in a document. Clockwise rolling corresponds to scrolling down. (c) The original shape of a Hermite curve. Blue points and arrows represent the positions and tangent vectors of control points. (d) The user moves a control point, rolls the pen clockwise, and applies more pressure. The tangent vector is moved, rotated and lengthened correspondingly.

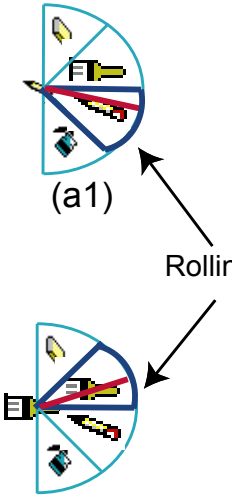

(b1)

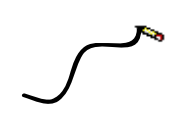

(a2)

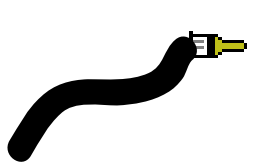

(b2)
Figure 13. Mode selection. (a1) Roll the pen within $\left[45^{\circ}, 0^{\circ}\right]$ to select the "pencil" tool. (a2) The pencil tool has been selected by pen rolling. (b1) Roll the pen within $\left[-45^{\circ}, 0^{\circ}\right]$ to select the "brush" tool. (b2) The brush tool has been selected by pen rolling 


\section{INFORMAL USER FEEDBACK}

In order to gather early user feedback, we asked six users (five male and one female) ages 22-36 to try the rollingbased prototype applications. Each participant was shown the six rolling-based interactions described above (i.e., rotating puzzle pieces, animating a graphic, map navigation, scrolling down/up in a document, editing a curve, and switching drawing tools), and then asked to freely and extensively try out these six techniques. Each usability session lasted 40 to 50 minutes. We observed participants' behaviors, and informally interviewed them about their opinions and suggestions.

All participants felt comfortable rolling the pen within the usable range. Two participants reported that they could roll the pen by a larger angle, suggesting that we enlarge the usable range. Only one person accidently invoked the rolling-based interaction techniques, doing so twice when he was creating a simple drawing with the pen. While these observations provide preliminary usability evidence that rolling speed and rolling angle thresholds are a reliable means for distinguishing incidental rolling from intentional rolling, and that our empirically determined thresholds are reasonable, they also indicate that it may be useful to allow individual adjustments of the thresholds for advanced users.

All participants were able to quickly learn the rolling-based techniques. One participant commented that "it's pretty straight forward" and that he could "spend little time to learn." Limiting activation of the rolling-based techniques only to intentional rolling was broadly welcomed. Four participants commented that this added useful functions to a pen without affecting existing ones. All of the participants liked using rolling to rotate an object. One commented that the object moved and rotated as if attached to the pen tip. Another said that it felt "natural and intuitive" to rotate an object by rolling. Associating rolling with mouse wheel actions [20] was unanimously welcomed. One participant reported that "now the pen can be as powerful as a mouse". The visualization method which shows the mapping between rolling direction and scrolling direction worked well. No participant got confused about the mapping. Regarding the drawing tools selection application (Figure 13), one user commented that "it is pretty cool, like using a Swiss Army Knife". One participant expressed the desire to use this technique in Adobe Photoshop, to reduce moving the pen back and forth for switching tools.

The one major complaint was regarding the human ability in controlling multiple degree-of-freedoms of a pen. One participant reported that it was difficult to simultaneously control magnitude, direction and position of a control vector in the curve editing application (Figure 12c\&d). He manipulated each parameter separately, even though simultaneous control of these parameters is allowed. In tool switching applications, because more time is required to roll a pen a bigger angle, two participants suggested mapping $\left[-45^{\circ}, 0^{\circ}\right]$, and $\left[0^{\circ}, 45^{\circ}\right]$ ranges with the most frequently used tools.

\section{CONCLUSIONS and FUTURE WORK}

We have presented two experiments to investigate incidental pen rolling made by users when performing regular drawing and writing tasks, as well as users' ability to control intentional rolling. Building on the experimental results, we present an exploration of the design space of rolling-based interaction techniques.

Experiment 1 indicates that we can reliably identify incidental pen rolling, thus making pen rolling a plausible input modality for augmenting pen-based interaction. One can identify incidental rolling when the rolling speed is between $-30 \%$ and $30 \%$ or the rolling angle is between $-10^{\circ}$ and $10^{\circ}$. Furthermore, these ranges are the same for both left-handed and right-handed users, and so handedness need not be taken into account when classifying rolling as incidental or intentional.

Experiment 2 reveals that the usable range of rolling angle is $90^{\circ}$ in both the clockwise and counterclockwise directions. Within this range, targets with an angular span of as little as 10 degrees (easily discriminablee rotation) can be effectively specified by the user.

Using these empirically determined thresholds, our exploration of the design space of pen-rolling interactions focused on three application areas where pen rolling might be beneficially employed: enhanced stimulus-response compatibility in rotation tasks [7], multi-parameter input, and simplified mode selection. Informal user feedback on our prototype implementations indicates that these techniques are generally discoverable and usable.

While these prototypes of rolling-based interaction techniques are promising, they are still in the very early stages of design. They clearly require fine-tuning and formal evaluation. Another important direction of study is to explore the effect of visual feedback on how users control rolling. An understanding of this effect will allow for the creation of more effective widgets designed for rolling-based interaction. Visualizations should be considered for both purely rolling-based techniques, as well as hybrid methods that combine position with rolling angle. Furthermore, additional research is required into human ability to concurrently control multiple degrees of freedom of the pen. Such an investigation should likely also explore the differences between concurrent control and rapid switching between different degrees of freedom.

\section{ACKNOWLEDGEMENTS}

We would like to thank John Hancock, Noah Lockwood, the members of DGP lab and the participants of our studies. 


\section{REFERENCES}

1. Wacom Technologies: www.wacom.com

2. Vicon: www.vicon.com

3. Accot, J. and Zhai, S. (1997). Beyond Fitts' Law: Models for trajectory-based HCI tasks. ACM CHI Conference on Human Factors in Computing Systems, p. 295-302.

4. Balakrishnan, R., Baudel, T., Kurtenbach, G., and Fitzmaurice, G. (1997). The rockin'mouse: integral 3D manipulation on a plane. ACM CHI Conference on Human Factors in Computing Systems, p. 311-318.

5. Buxton, W. and Myers, B. (1986). A study in twohanded input. ACM CHI Conference on Human Factors in Computing Systems, p. 321-326.

6. Fitts, P.M. (1954). The information capacity of the human motor system in controlling the amplitude of movement. Journal of Experimental Psychology, 47. p. 381-391.

7. Fitts, P.M. and Seeger, C.M. (1953). S-R compatibility: spatial characteristics of stimulus and response codes. Journal of Experimental Physiology, 47. p. 381-391.

8. Fitzmaurice, G., Khan. A., Pieke, R., Buxton, B., Kurtenbach, G. (2003) Tracking Menus. ACM UIST Symposium on User Interface Software and Technology, p. 71-80.

9. Froehlich, B., Hochstrate, J., Skuk, V., and Huckauf, A. (2006). The globefish and the globemouse: two new six degree of freedom input devices for graphics applications. ACM CHI Conference on Human Factors in Computing Systems, p. 191-199.

10. Hinckley, K., Cutrell, E., Bathiche, S., and Muss, T. (20020). Quantitative analysis of scrolling techniques. ACM CHI Conference on Human Factors in Computing Systems, p. 65-72.

11. Hinckley, K., Pausch, R., Proffitt, D., Patten, J., and Kassell, N. (1997). Cooperative bimanual action. $A C M$ CHI Conference on Human Factors in Computing Systems, p. 27-34.

12. Li, Y., Hinckley, K., Guan Z., Landay, J. (2005). Experimental analysis of mode switching techniques in pen-based user interfaces. ACM CHI Conference on Human Factors in Computing Systems, p. 461-470.

13. Liu, J., Pinelle, D., Sallam, S., Subramanian, S., and Gutwin, C. (2006). TNT: improved rotation and translation on digital tables. Graphics interface, p. 2532.

14. Jacob, R. (1994). Integrality and separability of input devices. ACM Transactions on Computer-Human Interaction, 1(1). p. 3-26.

15. Jones, L.A., Lederman, S.J. (2006) Human hand function. Oxford University Press, New York.

16. Miura, M., Kunifuji, S. (2006). RodDirect: twodimensional input with pen knob. Mobile HCI, p.113120

17. Napier, J.R. (1956). The prehensile movements of the human hand. Journal of Bones and Joint Surgery, 38, p. $902-913$.

18. Ramos, G., Boulos, M., and Balakrishnan, R. (2004). Pressure widgets. ACM CHI Conference on Human Factors in Computing Systems, p. 487-494.

19. Ramos, G., Cockburn, A., Balakrishnan, R., and Beaudouin-Lafon, M. (2007). Pointing lenses: facilitating pen input through visual-and motor-space magnification. ACM CHI Conference on Human Factors in Computing Systems, p. 757-766.

20. Suzuki, Y., Misue, K., and Tanaka, J. (2007). Stylus Enhancement to Enrich Interaction with Computers. 12th International Conference on Human-Computer Interaction. (HCI International 2007), LNCS 4551, p. $133-142$

21. Tian, F., Ao, X., Wang, H., Setlur, V., and Dai, G. (2007). The tilt cursor: enhancing stimulus-response compatibility by providing $3 \mathrm{~d}$ orientation cue of pen. ACM CHI Conference on Human Factors in Computing Systems, p. 303-306.

22. Wang, Y., C.L. MacKenzie, V.A. Summers, and K.S. Booth. (1998). The structure of object transportation and orientation ACM CHI Conference on Human Factors in Computing Systems, p. 312-319.

23. Zhai, S. (1995). Human performance in six degree of freedom input control. PhD thesis, Department of Mechanical and Industrial Engineering, University of Toronto.

24. Zhai, S., Smith, B. A., and Selker, T. (1997). Improving browsing performance: A study of four input devices for scrolling and pointing tasks. INTERACT, p. 286-292. 